\title{
The role of lipid-based nano delivery systems on oral bioavailability enhancement of fenofibrate, a BCS II drug: comparison with fast-release formulations
}

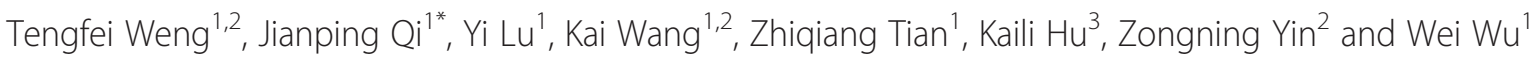

\begin{abstract}
The aim of this study was to compare various formulations solid dispersion pellets (SDP), nanostructured lipid carriers (NLCs) and a self-microemulsifying drug delivery system (SMEDDS) generally accepted to be the most efficient drug delivery systems for BCS II drugs using fenofibrate (FNB) as a model drug. The size and morphology of NLCs and SMEDDS was characterized by dynamic light scattering (DLS) and transmission electron microscopy (TEM). Their release behaviors were investigated in medium with or without pancreatic lipase. The oral bioavailability of the various formulations was compared in beagle dogs using commercial Lipanthy ${ }^{\circledR}$ capsules (micronized formulation) as a reference. The release of FNB from SDP was much faster than that from NLCs and SMEDDS in medium without lipase, whereas the release rate from NLCS and SMEDDS was increased after adding pancreatic lipase into the release medium. However, NLCs and SMEDDS increased the bioavailability of FNB to $705.11 \%$ and $809.10 \%$, respectively, in comparison with Lipanthy ${ }^{\oplus}$ capsules, although the relative bioavailability of FNB was only $366.05 \%$ after administration of SDPs. Thus, lipid-based drug delivery systems (such as NLCs and SMEDDS) may have more advantages than immediate release systems (such as SDPs and Lipanthyl ${ }^{\circledR}$ capsules).
\end{abstract}

Keywords: Fenofibrate, Solid dispersion, Nanostructured lipid carrier, Self-microemulsifying drug delivery system, Bioavailability

\section{Background}

According to the definition of the Biopharmaceutics Classification System (BCS) proposed by Amidon in 1995, both BCS II and IV drugs are poorly soluble in aqueous solution [1]. About $40 \%$ of new drug candidates identified by chemical screening are poorly soluble in water (BCS II or IV drugs), which greatly hinders their translation into the clinic [2]. However, the transmembrane permeation behavior of BCS II drugs is significantly different to that of BCS IV drugs. Generally, the apparent permeability coefficient (Papp) of BCS II drugs is greater than $10^{-6}$, whereas the Papp of BCS IV drugs is lower than $10^{-8}$ owing to various barriers such as low dissolution rate, low transmembrane permeability, efflux by transporter in the gut wall

\footnotetext{
* Correspondence: qijianping@fudan.edu.cn

${ }^{1}$ School of Pharmacy, Key Laboratory of Smart Drug Delivery of Ministry of Education, Fudan University, Shanghai 201203, PR China

Full list of author information is available at the end of the article
}

and first pass effect by metabolic enzymes [3]. To improve the oral bioavailability of these drugs, novel formulation technologies or drug delivery systems have emerged, including solid dispersion [4,5], nanocrystals [6], cyclodextrin inclusion [7,8], nanoemulsions [9], polymeric and lipidic nanoparticles (e.g. PLGA nanoparticles, solid lipid nanoparticles and nanostructure lipid carriers) [10-12]. These formulations can enhance oral absorption of drug molecules by improving dissolution in the gastrointenstinal tract (GIT) [6], facilitating adhesive interactions within the mucosa [13], increasing drug stability and improving lymphatic transport [14]. Nevertheless, different formulations have distinguishing features and facilitate absorption by distinct mechanisms. Solid dispersion and cyclodextrin inclusion improve the dissolution rate of poorly soluble drugs, but do not increase 
transmembrane permeability $[15,16]$. Nanocrystals are a fast-release system that has similar effects to those of solid dispersion and cyclodextrin inclusion [6], whereas nanoparticles can alter the permeability of the intestinal membrane by uptake of intact nanoparticles, facilitating adhesion and retention in the GIT and improving membrane fluidity, thus leading to increased absorption via the paracellular or transcellular route $[17,18]$. Furthermore, the fate of nanoparticles containing lipids in the GIT is different to to that of polymer nanoparticles. Digestion products of lipid nanoparticles can solubilize lipophilic drugs and the presence of endogenous bile salts may alter the intrinsic permeability of the intestinal membrane $[19,20]$. Although each drug delivery system may be recognized to improve oral bioavailability of poorly soluble drugs, we aimed to identify the optimal formulation technology for delivery of BCS II or IV drugs. Therefore, in this study, we first compared the bioavailability of different drug delivery systems loaded with the BCS II drug, fenofibrate (FNB).

FNB, a widely used hypolipidemic agent, is a typical BCS II drug. Due to its very low solubility in aqueous solution, the oral bioavailability is limited by slow dissolution [21]. In the clinic, micronized FNB (Lipanthyl ${ }^{\circ}$ capsules) showed significantly improved dissolution and enhanced oral bioavailability. More recently, various oral carrier systems were developed to increase oral absorption of FNB, including solid dispersion [4], a self-microemulsifying drug delivery system [22], liposome containing bile salts [23], mesoporous carbon [24], nanocystals [21] and lipidbased formulations [25]. Although these systems successfully increase the oral bioavailability of FNB, the optimal formulation remains to be identified by comparing the oral bioavailability of FNB after administration of different formulations.

Herein, the oral bioavailability of FNB-loaded into the lipid-based delivery systems, SMEDDS and NLCs, was compared with that of fast-release FNB SDPs and micronized Lipanthyl ${ }^{\bullet}$ capsules in beagle dogs.

\section{Results and discussion}

\section{Preparation and characterization of SDP, NLCs and SMEDDS}

FNB-loaded SDP, NLCs and SMEDDS were prepared successfully. Since FNB-loaded SDPs and NLCs were prepared according to our previous study $[25,26]$, the detailed characterization data are not shown in this report. The particle size of the obtained NLCs was $93.76 \pm 1.25 \mathrm{~nm}$ (polydispersity index (PDI), $0.222 \pm 0.014$ ), the zeta potential was $-29.1 \pm 4.1 \mathrm{mV}$ and the entrapment efficiency was approximately $96.66 \pm 1.01 \%$, which are similar to the values obtained in our previous study [25]. FNB-loaded SMEDDS were microemulsified in deionized water, $\mathrm{pH} 1.2 \mathrm{HCl}$

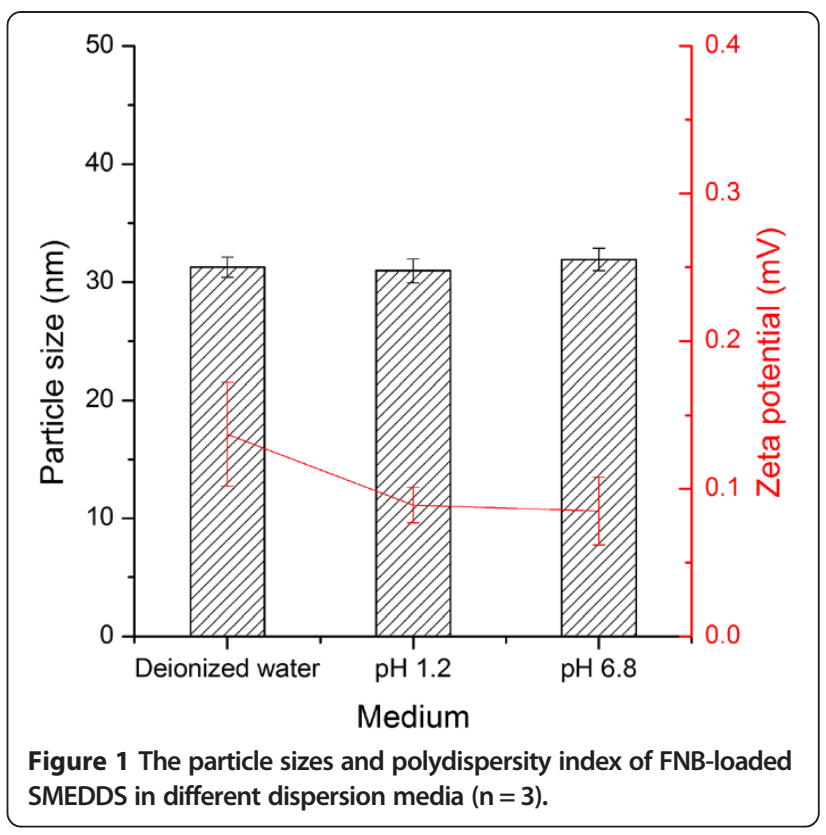

solution and pH 6.8 PBS immediately; the particle size and PDI are shown in Figure 1.

\section{Morphology}

Transmission electron microscopy (TEM) was employed to observe the morphology of NLCs and microemulsions formed by SMEDDS; micrographs are presented in Figure 2. The NLCs (Figure 2A) were spherical in shape and approximately $100 \mathrm{~nm}$ in size. TEM showed that the SMEDDS were emulsified in deionized water to generate uniform spherical microemulsion droplets approximately $20 \mathrm{~nm}$ in size (Figure $2 \mathrm{~B}$ ).

\section{In vitro release}

The profiles of FNB release from the three test formulations and the reference (commercial Lipanthyl ${ }^{\circ}$ capsules containing micronized FNB) in the media with or without pancreatic lipase are shown in Figure 3. FNB was released rapidly from Lipanthyl ${ }^{\circ}$ capsules and SDPs, with a cumulative release of more than $80 \%$ within $60 \mathrm{~min}$. Only about $25 \%$ of the FNB was released from SMEDDS within $24 \mathrm{~h}$ and even less (about 12\%) from the NLCs. However, pancreatic lipase changed the dissolution behavior of SMEDDS and NLCs. In release medium containing pancreatic lipase, the release of FNB from SDPs and Lipanthyl $l^{\circ}$ capsules was not altered significantly compared with that in medium without lipase. Nevertheless, the release from SMEDDS and NLCs was evidently improved, with more than $60 \%$ and $40 \%$ of FNB released from SMEDDS and NLCs in $24 \mathrm{~h}$, respectively. The similarity factor $\left(f_{2}\right)$, which is recommended by FDA for evaluation of the similarity of release profiles [27], was 

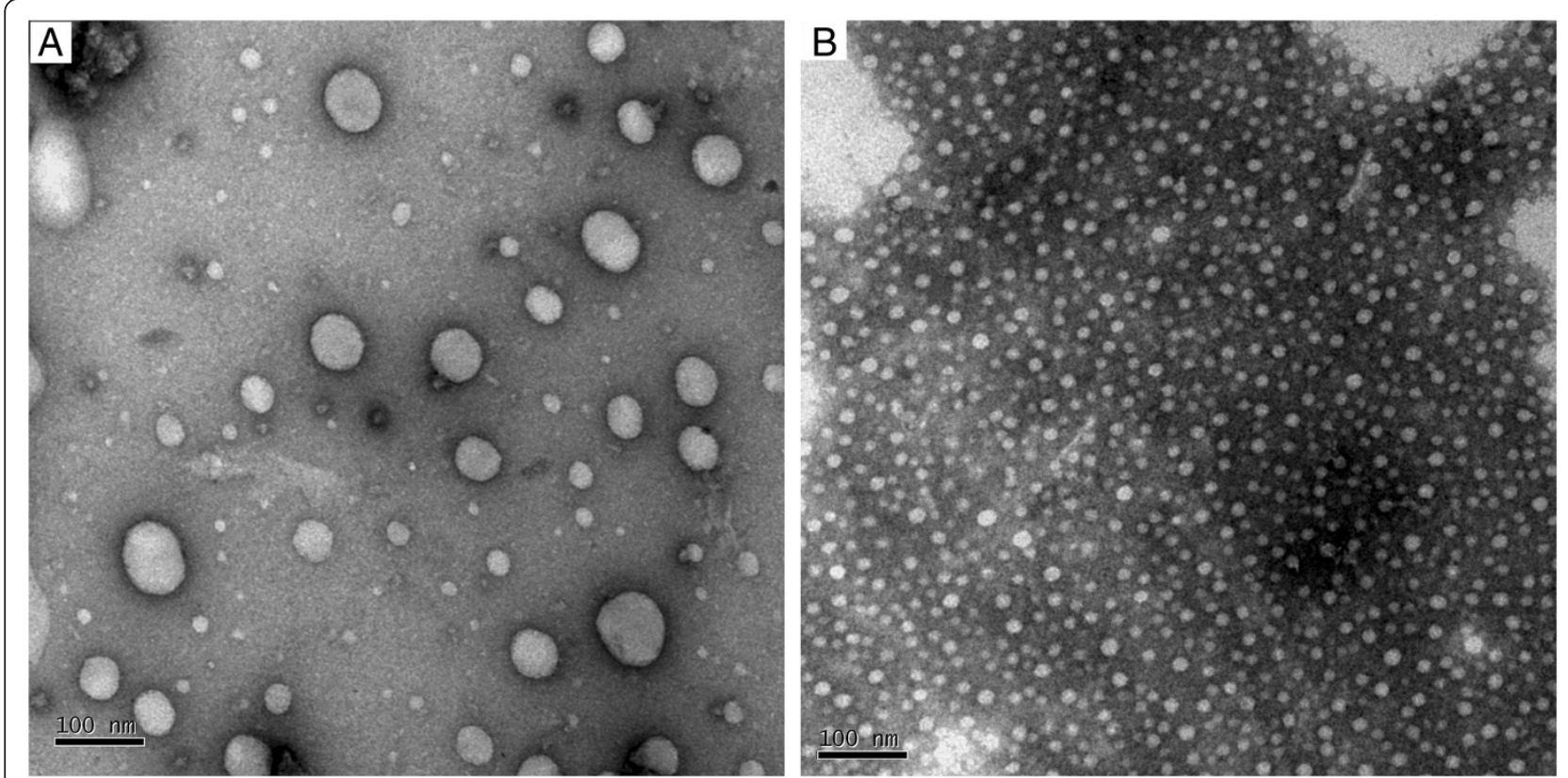

Figure 2 Morphology of FNB-loaded NLCs (A) and microemulsions droplets formed by SMEDDS (B) observed by transmission electron microscopy.

employed to evaluate the influence of lipase on FNB release according to the following formula:

$$
f_{2}=50 \times \lg \left(\sqrt{\left[1+\left(\frac{1}{n}\right) \sum_{i=1}^{n}\left(x_{t i}-x_{r i}\right)^{2}\right]} \times 100\right)
$$

where $x_{t i}$ and $x_{r i}$ are the cumulative release of time interval " $i$ " in release medium with and without pancreatic lipase, respectively, and $\mathrm{n}$ is the time interval. When $f_{2}$ is between 50 and 100 , the variation in every observation point between the two release profiles is not more than $10 \%$, which is considered to represent similarity. If $f_{2}<50$, the two release profiles are considered to be dissimilar. The $f_{2}$ of the four formulations are displayed in Table 1. The release profiles of FNB from Lipanthyl ${ }^{\circ}$ capsules and SDPs were not altered by the addition of lipase to the release medium, although the release profiles from SMEDDS and NLCs in two release media differed considerably, which indicates that intestinal lipase is important to the release of poorly
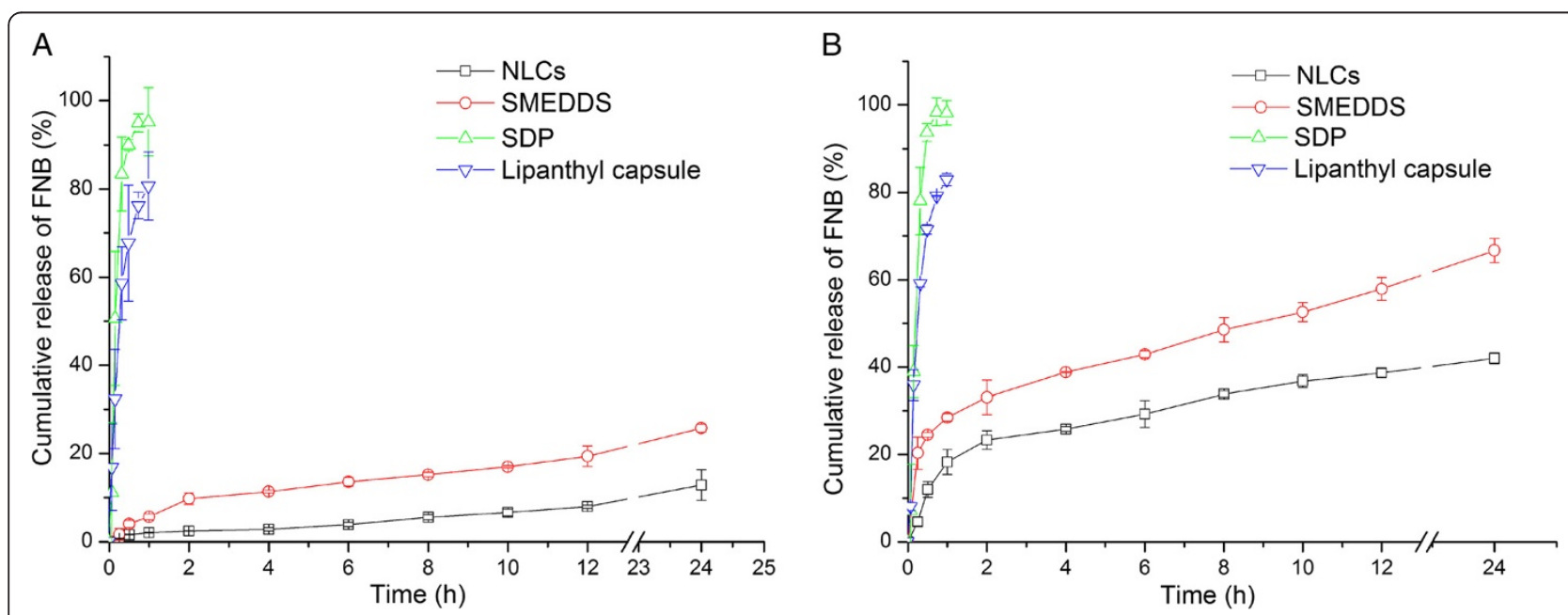

Figure 3 In vitro release profiles of FNB from SDP, NLCs, SMEDDS and Lipanthyl capsules in release media without (A) or with (B) pancreatic lipase $(n=3)$. 
Table 1 The $f_{2}$ values of release profiles of FNB in release media with or without pancreatic lipase

\begin{tabular}{lllll}
\hline Formulations & Lipanthy $^{\bullet}$ capsules & SDP & SMEDDS & NLCs \\
\hline$f_{2}$ & 67.1 & 60.7 & 36.5 & 42.4 \\
\hline
\end{tabular}

soluble drugs from lipid-based drug delivery systems, such as SMEDDS and NLCs.

\section{Oral bioavailability}

To illustrate the optimal formulation for BCS II drugs, oral bioavailability of FNB-loaded SDPs, NLCs and SMEDDS in beagle dogs were compared. The mean plasma FNB concentration versus time plots of the four formulations are shown in Figure 4 and the pharmacokinetic parameters obtained by analysis based on statistical moment theory are shown in Table 2.

After oral gavage administration of the three FNB formulations to beagle dogs, the $\mathrm{C}_{\max }$ and AUC of all of the formulations were improved compared with those of Lipanthy $l^{\circ}$ capsules. NLCs and SMEDDS in particular exhibited enhanced absorption compared with SDPs. The relative bioavailability of NLCs and SMEDDS were $705.11 \%$ and $809.10 \%$, respectively, compared with Lipanthyl $^{\circ}$ capsules, while that of SDPs was only $366.05 \%$. Compared with Lipanthyl $1^{\circ}$ capsules, the $T_{\max }$, MRT and $t_{1 / 2}$ of fenofibric acid showed no significant changes after oral administration of all three formulations.

Theoretically, the oral bioavailability of BCS II drugs is restricted mainly by poor dissolution in the GIT. Generally speaking, the oral bioavailability of BCS II drugs is improved greatly if the in vitro dissolution is enhanced [28]. Therefore, micronization, nanosuspension, solid dispersion and cyclodextrin inclusion are widely used to

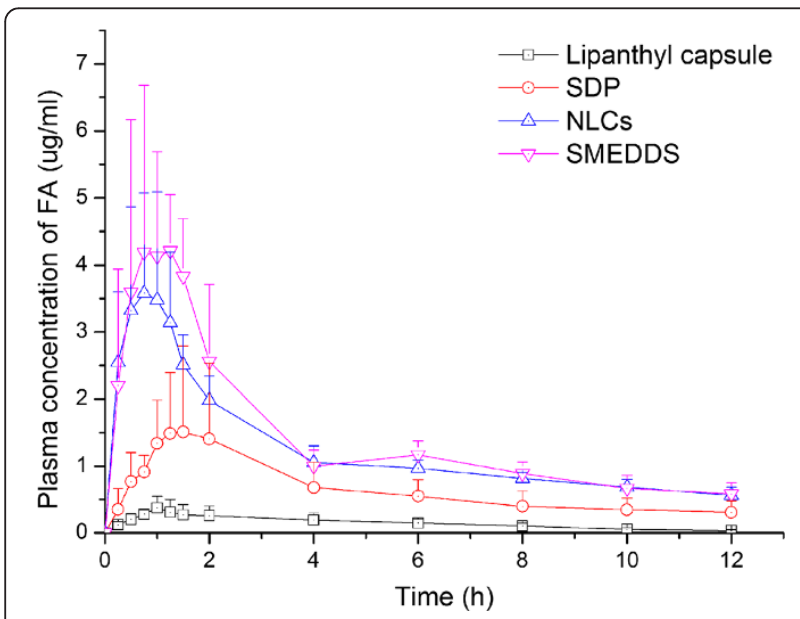

Figure 4 Mean plasma concentration-time profiles of fenofibric acid in beagle dogs after oral administration of FNB-loaded SDP, NLCs, SMEDDS and Lipanthyl ${ }^{\circledR}$ capsules $(n=6)$. improve the oral bioavailability of BCS II drugs [28]. Previous in vitro and in vivo evaluations of the reference, Lipanthy $l^{\circ}$ capsules, which are a product of micronized FNB and nanosuspensions of FNB suggested the FNB is rapidly released from Lipanthyl ${ }^{\circ}$ capsules, SDPs and nanosuspensions, and that SDPs or nanosuspensions improve the oral bioavailability of FNB compared with that of the Lipanthyl ${ }^{\circ}$ capsules [29]. Similar dissolution does not lead to the same oral absorption, which may be due to the various influences of the GIT contents on dissolution of drugs from the different formulations.

Although FNB was released very slowly and in small amounts from lipid-based drug delivery systems, such as NLCs and SMEDDS, the cumulative release of FNB increased with introduction of lipase. Pancreatic lipase, bile salts and phospholipids are continuously secreted into the GIT. Lipid-based drug delivery systems are digested by pancreatic lipase to form secondary structures, such as mixed micelles, cubic or hexagonal nanoparticles and vesicular carriers [30]. Therefore, drugs can be solubilized in these secondary derivatives when lipidbased formulations are digested [31]. SDPs, nanosuspensions or micronized drugs significantly increase drug dissolution, but oral absorption is promoted only by the original absorption pathways of the drug itself. Nevertheless, lipid-based drug delivery systems may enhance the absorption of drugs through diverse pathways [32].

On the one hand, NLCs and SMEDDS can adhere to the gut wall to increase retention time in GIT. The particle sizes of NLCs and SMEDDS were below $100 \mathrm{~nm}$, which endows them with a massive specific surface area and facilitates the adhesion of nanoparticles by the mucus layer $[33,34]$. On the other hand, many reports have suggested that the digestion of lipid-based drug delivery systems in the GIT is the most important factor required to enhance the absorption of poorly soluble drugs [35-37]. Exogenous lipids stimulate the secretion of biliary lipids (bile salts, phospholipids and cholesterol), which combine with lipid digestion products to generate a series of colloidal species, including micelles, mixed micelles, vesicles and emulsion droplets [38]. These colloidal species provide a reservoir of solubilized drug at the absorptive site and generate the concentration gradient required to drive improved absorption. Luminal amphiphiles, such as bile salts, may also enhance the solubilization of drugs by improving wetting at concentrations below the critical micellar concentration [39]. Thus, the drug concentration increases during the digestion process, which improves the transport across nonstirred water layers and then the bio-membranes. In addition, fatty acids and monoglycerides produced during digestion increase the fluidity and permeability of membranes due to their surface activity, which is also an important factor in enhancing drug absorption [40]. Furthermore, lymphatic transport can also increase the oral bioavailability 
Table 2 The main pharmacokinetic parameters of fenofibric acid in beagle dogs after oral administration of FNB-loaded SDP, NLCs, SMEDDS and Lipanthyl ${ }^{\circledR}$ capsules $(\mathbf{n}=6)$

\begin{tabular}{lllll}
\hline Parameters & Lipanthyl $\left.\right|^{\circledR}$ capsules & SDP & NLCs & SMEDDS \\
\hline$C_{\max }(\mu \mathrm{g} / \mathrm{mL})$ & $0.41 \pm 0.17$ & $1.82 \pm 1.18^{*}$ & $3.87 \pm 1.40^{* \#}$ & $5.31 \pm 1.18^{* \# \mathbf{~}}$ \\
$T_{\max }(\mathrm{h})$ & $1.04 \pm 0.29$ & $1.13 \pm 0.59$ & $0.92 \pm 0.34$ & $0.96 \pm 0.43$ \\
$t_{1 / 2}(\mathrm{~h})$ & $3.40 \pm 1.55$ & $9.58 \pm 7.64$ & $8.09 \pm 3.25^{*}$ & $6.25 \pm 2.15^{*}$ \\
$M R T_{(0-t)}(\mathrm{h})$ & $4.24 \pm 0.24$ & $4.45 \pm 0.18$ & $4.22 \pm 0.42$ & $4.00 \pm 0.28$ \\
$A \cup C_{(0-t)}\left(\mu \mathrm{g} / \mathrm{mL}^{*} \mathrm{~h}\right)$ & $2.13 \pm 0.69$ & $7.81 \pm 4.36^{*}$ & $15.04 \pm 2.34^{* \#}$ & $17.26 \pm 3.43^{* \#}$ \\
$F_{1}(\%)$ & - & 366.05 & 705.11 & 809.10 \\
$F_{2}(\%)$ & - & - & 192.62 & 221.03 \\
$F_{3}(\%)$ & - & - & - & 114.75 \\
\hline
\end{tabular}

$\mathrm{F}_{1}, \mathrm{~F}_{2}$ and $\mathrm{F}_{3}$ are relative bioavailability of other formulations compared with Lipanthyl ${ }^{\varpi}$ capsules, SDP and NLCs, respectively.

${ }^{*} P<0.05$, compared with Lipanthyl ${ }^{\circledast}$ capsule.

$\# P<0.05$, compared with SDP.

$\Delta P<0.05$, compared with NLCs.

of lipophilic drugs [41]. Lipid vehicles may enhance lymphatic transport of lipophilic compounds by simulating the production of chylomicrons. Lipophilic drugs enter the lymphatic system in association with the triglyceride core of the chylomicrons [42]. The digestibility of the vehicle is a prerequisite for the production of the fatty acids necessary to drive chylomicron production [43].

\section{Conclusion}

FNB-loaded SDPs, NLCs and SMEDDS were prepared and their in vitro and in vivo properties were compared. SDPs significantly increased the release of FNB in medium with and without lipase, which is similar to the characteristics of Lipanthyl ${ }^{\circ}$ capsules. Pancreatic lipase improved the release of FNB from NLCs and SMEDDS remarkably. However, the oral bioavailability of FNB after administration of NLCs and SMEDDS was significantly higher than that of SDP and Lipanthyl ${ }^{\circ}$ capsules $(P<0.05)$. Therefore, lipid-based drug delivery systems (such as NLCs and SMEDDS) are more advantageous than the other drug delivery systems (solid dispersion or micronization) for BCS II drugs, due to the multiple absorption enhancement mechanisms. Lipidbased drug delivery systems may be an excellent candidate for oral formulation of insoluble drugs.

\section{Methods}

\section{Materials}

FNB was purchased from Nhwa Pharmaceutical Group (Xuzhou, China). Polyvinylpyrrolidone (PVP K30) was kindly gifted from China Division, ISP Chemicals Co. (Shanghai, China). Non-pareil pellets (Suglets ${ }^{\circ}$ sugar spheres PF101, 710-850 $\mu \mathrm{m}$ in diameter) were provided by NP Pharm (Bazainville, France). Precirol ATO 5 and Captex 100 were kindly provided by Gattefossé Co. (Saint Priest, Cedex, France) and Abitec Co. (OH, USA), respectively. Polysorbate 80 (Tween-80) was supplied by Shenyu Pharmaceutical and Chemical Co., Ltd (Shanghai). Oleoyl macrogolglycerides (Labrafil $\mathrm{M}^{\circ} 1944 \mathrm{CS}$ ) and diethylene glycol monoethyl ether (Transcutol $\mathrm{P}^{\circ}$ ) were purchased from Gattefossé Co. Ethoxylated castor oil (Cremophor ${ }^{\circ}$ EL) was obtained from BASF Corporation (Ludwigshafen, Germany). HPLC grade methanol and acetonitrile were purchased from Tedia (Carson City, NV, USA). Deionized water was prepared using a Milli-Q purification system (Millipore, Billerica, MA, USA). All other chemicals were of analytical grade and were used as received.

\section{Preparation of FNB-loaded delivery systems \\ Preparation of solid dispersion pellets (SDP)}

FNB-loaded SDPs were prepared using a Mini-Glatt fluidbed coater (Wurster insert; Glatt GmbH, Binzen, Germany) based on previously established procedures [26]. FNB, PVP K30 and sodium dodecyl sulfate (SDS) (4:3:3, w/w) were dissolved in $90 \%$ ethanol. The resulting solution was sprayed through a nozzle $(0.5 \mathrm{~mm}$ in diameter) onto the fluidized non-pareil pellets to obtain a coating weight gain of approximately $100 \%$. The detailed operating conditions were as follows: product temperature, $35^{\circ} \mathrm{C}-40^{\circ} \mathrm{C}$; air flow rate, $97-103 \mathrm{~m}^{3} / \mathrm{h}$; spray rate, $0.6 \mathrm{~mL} /$ minute; atomizing air pressure, 1.4-1.5 bar. The pellets were further dried for 15 min after coating completion.

\section{Preparation of nanostructured lipid carriers (NLCS)}

The NLC suspension was prepared by the meltingemulsification method according to our previously described procedures [25]. Briefly, $1.14 \mathrm{~g}$ solid lipid phase (Precirol ATO 5) and $0.48 \mathrm{~g}$ liquid lipid phase (Captex 100) were melted at $80^{\circ} \mathrm{C}$ and mixed. Then $60 \mathrm{mg}$ FNB was dissolved in the lipid mixture. The melted mixture was then dispersed in a hot $\left(80^{\circ} \mathrm{C}\right)$ aqueous solution $(30 \mathrm{~mL})$ containing Tween-80 $(2 \%, \mathrm{w} / \mathrm{v})$ for $3 \mathrm{~min}$ at a rate of 8,000 rpm using a high-speed Ultra Turrax blender (QilinBeier, Jiangsu, China) to produce the coarse emulsion. 
Subsequently, the coarse emulsion was homogenized using a high-pressure homogenizer (Microfluids, Nano DeBee, USA) for three cycles under 20,000 psi. The obtained hot NLC suspension was cooled to room temperature for use in further investigations.

\section{Preparation of self-microemulsifying drug delivery systems (SMEDDS)}

The formulation of FNB-loaded SMEDDS was performed according to previously described methods with modifications [44]. Briefly, FNB, Labrafil M 1944 CS (oil phase), Cremophore EL (surfactant) and Transcutol P (co-surfactant) were mixed in ratio of 40/520/585/195 (w/w). The obtained SMEDDS was stored at $4^{\circ} \mathrm{C}$ before use.

\section{Measurement of particle size}

Particle size was measured by Zetasizer $\mathrm{Nano}^{\circ}$ (Malvern Instruments, Malvern, UK) equipped with a $4 \mathrm{~mW} \mathrm{He}-\mathrm{Ne}$ laser $(633 \mathrm{~nm})$ at $25^{\circ} \mathrm{C}$. The NLC suspension was diluted 15 -fold with deionized water before measurement. The particle size of SMEDDS was determined after microemulsification in deionized water. Three measurements were conducted, and the number of runs in each measurement was automatically determined by the software.

\section{Transmission electron microscopy (TEM)}

TEM was used to characterize the morphology of NLCs and SMEDDS. Prior to examination, microemulsion drops were obtained by emulsifying SMEDDS in deionized water. The NLCs suspension and microemulsion droplets were then placed on copper grids and negatively stained with $2 \%(\mathrm{w} / \mathrm{v})$ phosphotungstic acid for $5 \mathrm{~min}$ at room temperature. Finally, the grids bearing NLCs and microemulsion droplets were observed with a JEM-1230 transmission electron microscope (JEOL, Tokyo, Japan).

\section{Entrapment efficiency of NLCs}

The entrapment efficiency of NLCs was determined by ultrafiltration. Briefly, $0.4 \mathrm{~mL}$ NLCs was added to an ultrafiltration tube $(100 \mathrm{kD})$ and then centrifuged for $10 \mathrm{~min}$ at $4,000 \times g$. The concentrations of FNB in the filtrate $\left(C_{\text {free }}\right)$ was determined by HPLC directly. The concentration of FNB original NLCs $\left(C_{\text {total }}\right)$ were determined as following method. Briefly, $0.4 \mathrm{~mL}$ of NLCs suspension was dissolved in $100 \mathrm{~mL}$ methanol. The FNB released into methanol from NLCs rapidly with the help of ultrasound. After ultrasound treatment of $20 \mathrm{~min}$, the mixed solution was centrifuged for $10 \mathrm{~min}$ under $10,000 \times$ g. The supernatant was injected into HPLC to determine $C_{\text {total }}$. The entrapment efficiency (EE) was calculated according to the following equation.

$$
\mathrm{EE}=\frac{C_{\text {total }}-C_{\text {free }}}{C_{\text {total }}} \times 100 \%
$$

\section{Release test}

The release test was performed in a ZRS-8G dissolution tester (Tianda Tianfa Technology Co. Ltd, Tianjin, China) according to the Chinese Pharmacopoeia (2010) Appendix Method III. To clarify the effect of lipase on the release of lipid formulations (NLCs or SMEDDS), we selected two different release media; phosphate balanced saline ( $\mathrm{pH}$ 6.8) containing 2\% Cremophor EL with or without pancreatic lipase $(100 \mathrm{IU} / \mathrm{mL})$. Four formulations (containing $3 \mathrm{mg} \mathrm{FNB}$ ) were added into $100 \mathrm{~mL}$ release medium that was thermostatically maintained at $37 \pm 0.5^{\circ} \mathrm{C}$ and stirred at a revolution speed of $100 \mathrm{rpm}$. SDP was sealed into hard gelatin capsules. Samples of $0.5 \mathrm{~mL}$ were withdrawn at specific time intervals and immediately ultrafiltered (Millipore, $100 \mathrm{kD}$ ) at 4,000 $\times g$ for $10 \mathrm{~min}$. The ultrafiltrate was assayed for FNB by HPLC as described later in the text.

\section{Bioavailability study}

The bioavailability of SDPs, NLCs and SMEDDS containing FNB was evaluated in beagle dogs using commercially available Lipanthyl $1^{\bullet}$ capsules (micronized FNB, Solvay Pharma) as a reference. Beagle dogs (adult males, $15.0 \pm 0.5 \mathrm{~kg}$ ) used in the experiments received care in compliance with the Principles of Laboratory Animal Care and the Guide for the Care and Use of Laboratory Animals. Experiments followed protocols approved by the Fudan University Institutional Animal Care and Use Committee.

Four formulations were administered to the dogs by oral gavage at an equivalent dose of $3 \mathrm{mg} / \mathrm{kg}$ FNB. Blood samples $(1.5 \mathrm{~mL})$ were then collected into heparinized tubes at designated time intervals: $0.25,0.5,0.75,1,1.25$, $1.5,2,4,6,8,10$ and $12 \mathrm{~h}$. Plasma was separated by centrifugation for $10 \mathrm{~min}$ at $4,000 \times g$ and frozen at $-18^{\circ} \mathrm{C}$ for subsequent analysis. FNB, as a prodrug, is rapidly metabolized into its major active metabolite, fenofibric acid (FA), after absorption. Intact FNB cannot be detected in the plasma after oral administration; therefore, pharmacokinetic evaluation of FNB was based on the quantification of FA in the plasma [45]. FA in dog plasma was extracted by liquid-liquid extraction procedures established in our previous study and the concentration of FA was determined by HPLC [23].

Pharmacokinetic parameters were calculated by noncompartmental analysis based on statistical moment theory using DAS professional software version 2.0 (Anhui, China). The pharmacokinetic parameters, such as peak plasma concentration $\left(C_{\max }\right)$, the time to maximum 
plasma concentration $\left(T_{\max }\right)$, and the area under the concentration-time curve between 0 and $12 \mathrm{~h}\left(A U C_{0-12}\right)$ were determined.

\section{HPLC analysis}

Both in vitro and in vivo samples were determined by HPLC system (Agilent 1260 series, California, USA) comprising an auto sampler, a pump, a column oven, and a tunable ultraviolet detector. The analytical column was a C18 column (Diamonsil ${ }^{\circ}, 5 \mu \mathrm{m}, 4.6 \mathrm{~mm} \times 250 \mathrm{~mm}$, Dikma, China) guarded with a refillable precolumn (C18, $2.0 \mathrm{~mm} \times 20 \mathrm{~mm}$, Alltech, USA). The flow rate was $1.0 \mathrm{~mL} / \mathrm{min}$. The UV-detector was set at a wavelength of $287 \mathrm{~nm}$. The column temperature was set to $40^{\circ} \mathrm{C}$. In terms of in vitro determination of FNB, the mobile phase consisted of methanol and deionized water mixed at a ratio of 90/10 (v/v). However, the mobile phase was composed of a mixture of methanol, water and 10\% phosphoric acid $(70 / 30 / 1, \mathrm{v} / \mathrm{v} / \mathrm{v})$ for in vivo determination of FA. Indomethacin $(10 \mu \mathrm{g} / \mathrm{mL})$ was used as an internal standard [23].

\section{Statistical analysis}

All data were expressed as mean \pm standard deviation (SD). One-way ANOVA followed by Tukey's test was performed to assess the statistical significance of differences. Results with $P<0.05$ were considered statistically significant.

\section{Competing interests}

The authors declare that they have no competing interests.

\section{Authors' contributions}

TW performed the majority of the experiments and wrote the manuscript with $J Q$ and $W W . J Q, W W, Y L$ and $Z Y$ designed the overall project and aided with data interpretations. KW and ZT assisted with the animal experiments. $\mathrm{KH}$ assisted to perform the transmission electron microscopy. All authors read and approved the final manuscript.

\section{Acknowledgements}

We are grateful for the financial support from Shanghai Commission of Science and Technology (14JC1490300, 11 nm0506700). Dr. Wu would also want to thank Shanghai Commission of Education (10SG05) and Ministry of Education (NCET-11-0114) for personnel fostering support.

\section{Author details}

'School of Pharmacy, Key Laboratory of Smart Drug Delivery of Ministry of Education, Fudan University, Shanghai 201203, PR China. ${ }^{2}$ West China School of Pharmacy, Sichuan University, Chengdu, Sichuan 610041, PR China. ${ }^{3}$ Murad Research Center for Modernized Chinese Medicine, Shanghai University of Traditional Chinese Medicine, Shanghai 201203, PR China.

Received: 13 August 2014 Accepted: 13 September 2014 Published online: 24 September 2014

\section{References}

1. Amidon GL, Lennernas $H$, Shah VP, Crison JR: A theoretical basis for a biopharmaceutic drug classification: the correlation of in vitro drug product dissolution and in vivo bioavailability. Pharm Res 1995 , 12:413-420.

2. Chen H, Khemtong C, Yang X, Chang X, Gao J: Nanonization strategies for poorly water-soluble drugs. Drug Discov Today 2011, 16:354-360.
3. Benet LZ: The role of BCS (biopharmaceutics classification system) and BDDCS (biopharmaceutics drug disposition classification system) in drug development. J Pharm Sci 2013, 102:34-42.

4. Kawakami K, Zhang S, Chauhan RS, Ishizuka N, Yamamoto M, Masaoka Y, Kataoka M, Yamashita S, Sakuma S: Preparation of fenofibrate solid dispersion using electrospray deposition and improvement in oral absorption by instantaneous post-heating of the formulation. Int J Pharm 2013, 450:123-128.

5. Chen Z, Xie Y, Guan P, Xu Y, Qi J, Lu Y, Wu W: Enhanced oral bioavailability of all-trans-retinoic acid by 2 -hydroxypropyl- $\beta$-cyclodextrin inclusion complex pellets prepared by fluid-bed coating technique. Asian J Pharm Sci 2011, 6:202-207.

6. Ige PP, Baria RK, Gattani SG: Fabrication of fenofibrate nanocrystals by probe sonication method for enhancement of dissolution rate and oral bioavailability. Colloids Surf B: Biointerfaces 2013, 108:366-373.

7. Tokumura T, Muraoka A, Machida Y: Improvement of oral bioavailability of flurbiprofen from flurbiprofen/beta-cyclodextrin inclusion complex by action of cinnarizine. Eur J Pharm Biopharm 2009, 73:202-204.

8. Gao Y, Nishimura K, Hirayama F, Arima H, Uekama K, Schmid G, Terao K, Nakata D, Fukumi $\mathrm{H}$ : Enhanced dissolution and oral bioavailability of coenzyme Q10 in dogs obtained by inclusion complexation with $\gamma$ cyclodextrin. Asian J Pharm Sci 2006, 1:95-102.

9. Monteiro LM, Lione VF, Do Carmo FA, Do Amaral LH, Da Silva JH, Nasciutti LE, Rodrigues CR, Castro HC, De Sousa VP, Cabral LM: Development and characterization of a new oral dapsone nanoemulsion system: permeability and in silico bioavailability studies. Int J Nanomedicine 2012 7:5175-5182.

10. Ling G, Zhang P, Zhang W, Sun J, Meng X, Qin Y, Deng Y, He Z: Development of novel self-assembled DS-PLGA hybrid nanoparticles for improving oral bioavailability of vincristine sulfate by P-gp inhibition. J Control Release 2010, 148:241-248.

11. Das S, Chaudhury A: Recent advances in lipid nanoparticle formulations with solid matrix for oral drug delivery. AAPS PharmSciTech 2011 12:62-76.

12. Zhuang CY, Li N, Wang M, Zhang XN, Pan WS, Peng JJ, Pan YS, Tang X: Preparation and characterization of vinpocetine loaded nanostructured lipid carriers (NLC) for improved oral bioavailability. Int J Pharm 2010, 394:179-185.

13. Gupta PN, Khatri K, Goyal AK, Mishra N, Vyas SP: M-cell targeted biodegradable PLGA nanoparticles for oral immunization against hepatitis B. J Drug Target 2007, 15:701-713.

14. Beloqui A, Solinis MA, Delgado A, Evora C, Isla A, Rodriguez-Gascon A: Fate of nanostructured lipid carriers (NLCs) following the oral route: design, pharmacokinetics and biodistribution. J Microencapsul 2013, 31:1-8.

15. Sun $N$, Zhang $X, L u Y$, Wu W: In vitro evaluation and pharmacokinetics in dogs of solid dispersion pellets containing Silybum marianum extract prepared by fluid-bed coating. Planta Med 2008, 74:126-132.

16. Chen Z, Lu Y, Qi J, Wu W: Enhanced dissolution, stability and physicochemical characterization of ATRA/2-hydroxypropyl-betacyclodextrin inclusion complex pellets prepared by fluid-bed coating technique. Pharm Dev Technol 2013, 18:130-136.

17. Shaikh J, Ankola DD, Beniwal V, Singh D, Kumar MN: Nanoparticle encapsulation improves oral bioavailability of curcumin by at least 9-fold when compared to curcumin administered with piperine as absorption enhancer. Eur J Pharm Sci 2009, 37:223-230.

18. Sonaje K, Lin KJ, Tseng MT, Wey SP, Su FY, Chuang EY, Hsu CW, Chen CT, Sung HW: Effects of chitosan-nanoparticle-mediated tight junction opening on the oral absorption of endotoxins. Biomaterials 2011, 32:8712-8721.

19. Porter CJ, Kaukonen AM, Taillardat-Bertschinger A, Boyd BJ, O'Connor JM, Edwards GA, Charman WN: Use of in vitro lipid digestion data to explain the in vivo performance of triglyceride-based oral lipid formulations of poorly water-soluble drugs: studies with halofantrine. J Pharm Sci 2004, 93:1110-1121

20. Memvanga PB, Eloy P, Gaigneaux EM, Preat V: In vitro lipolysis and intestinal transport of beta-arteether-loaded lipid-based drug delivery systems. Pharm Res 2013, 30:2694-2705.

21. Zuo B, Sun Y, Li H, Liu X, Zhai Y, Sun J, He Z: Preparation and in vitro/ in vivo evaluation of fenofibrate nanocrystals. Int J Pharm 2013, 455:267-275. 
22. Patel AR, Vavia PR: Preparation and in vivo evaluation of SMEDDS (self-microemulsifying drug delivery system) containing fenofibrate. AAPS J 2007, 9:E344-E352.

23. Chen Y, Lu Y, Chen J, Lai J, Sun J, Hu F, Wu W: Enhanced bioavailability of the poorly water-soluble drug fenofibrate by using liposomes containing a bile salt. Int J Pharm 2009, 376:153-160.

24. Niu X, Wan L, Hou Z, Wang T, Sun C, Sun J, Zhao P, Jiang T, Wang S: Mesoporous carbon as a novel drug carrier of fenofibrate for enhancement of the dissolution and oral bioavailability. Int J Pharm 2013, 452:382-389.

25. Tian Z, Yi Y, Yuan H, Han J, Zhang X, Xie Y, Lu Y, Qi J, Wu W: Solidification of nanostructured lipid carriers (NLCs) onto pellets by fluid-bed coating: preparation, in vitro characterization and bioavailability in dogs. Powder Technol 2013, 247:120-127.

26. Tang N, Lai J, Chen YP, Lu Y, Wu W: Fenofibrate solid dispersion pellets prepared by fluid-bed coating: physical characterization, improved dissolution and oral bioavailability in beagle dogs. J Chin Pharm Sci 2009, 18:156-161

27. Fei Y, Kostewicz ES, Sheu MT, Dressman JB: Analysis of the enhanced oral bioavailability of fenofibrate lipid formulations in fasted humans using an in vitro-in silico-in vivo approach. Eur J Pharm Biopharm 2013 $85: 1274-1284$

28. Buckley ST, Frank KJ, Fricker G, Brandl M: Biopharmaceutical classification of poorly soluble drugs with respect to "enabling formulations". Eur J Pharm Sci 2013, 50:8-16.

29. Weng TF, Qi J, Lu Y, Yin Z, Wu W: Preparation and bioavailability study in beagle dogs of fenofibrate nanosuspension. Chin J Pharm 2014, 45:23-239.

30. Porter CJ, Pouton CW, Cuine JF, Charman WN: Enhancing intestinal drug solubilisation using lipid-based delivery systems. Adv Drug Deliv Rev 2008, 60:673-691.

31. Porter CJ, Trevaskis NL, Charman WN: Lipids and lipid-based formulations: optimizing the oral delivery of lipophilic drugs. Nat Rev Drug Discov 2007, 6:231-248.

32. Liu YO, Fan JM, Wang XQ, Zhang Q: Preparation of sorafenib selfmicroemulsifying drug delivery system and its relative bioavailability in rats. J Chin Pharm Sci 2011, 20:164-170.

33. He C, Yin L, Tang C, Yin C: Size-dependent absorption mechanism of polymeric nanoparticles for oral delivery of protein drugs. Biomaterials 2012, 33:8569-8578.

34. Wang $S, Y e T$, Zhang $X$, Yang $R$, Yi X: Myricetin loaded in microemulsion for oral drug delivery: formulation optimization, intestinal absorption in situ recirculation and in-vivo evaluation. Asian J Pharm Sci 2012, 7:293-300.

35. Dahan A, Hoffman A: The effect of different lipid based formulations on the oral absorption of lipophilic drugs: the ability of in vitro lipolysis and consecutive ex vivo intestinal permeability data to predict in vivo bioavailability in rats. Eur J Pharm Biopharm 2007, 67:96-105

36. Dahan A, Hoffman A: Rationalizing the selection of oral lipid based drug delivery systems by an in vitro dynamic lipolysis model for improved oral bioavailability of poorly water soluble drugs. J Control Release 2008, 129:1-10.

37. Porter CJ, Charman WN: In vitro assessment of oral lipid based formulations. Adv Drug Deliv Rev 2001, 50(Suppl 1):S127-S147.

38. Kossena GA, Charman WN, Wilson CG, O'Mahony B, Lindsay B, Hempenstal JM, Davison CL, Crowley PJ, Porter CJ: Low dose lipid formulations: effects on gastric emptying and biliary secretion. Pharm Res 2007, 24:2084-2096.

39. Luner PE, Vander Kamp D: Wetting behavior of bile salt-lipid dispersions and dissolution media patterned after intestinal fluids. J Pharm Sci 2001, 90:348-359

40. Pabla D, Akhlaghi F, Zia H: Intestinal permeability enhancement of levothyroxine sodium by straight chain fatty acids studied in MDCK epithelial cell line. Eur J Pharm Sci 2010, 40:466-472.

41. Khoo SM, Shackleford DM, Porter CJ, Edwards GA, Charman WN: Intestinal lymphatic transport of halofantrine occurs after oral administration of a unit-dose lipid-based formulation to fasted dogs. Pharm Res 2003, 20:1460-1465.

42. Gershkovich P, Hoffman A: Uptake of lipophilic drugs by plasma derived isolated chylomicrons: linear correlation with intestinal lymphatic bioavailability. Eur J Pharm Sci 2005, 26:394-404.
43. Caliph SM, Charman WN, Porter CJ: Effect of short-, medium-, and longchain fatty acid-based vehicles on the absolute oral bioavailability and intestinal lymphatic transport of halofantrine and assessment of mass balance in lymph-cannulated and non-cannulated rats. J Pharm Sci 2000 89:1073-1084

44. Hu L, Wu H, Niu F, Yan C, Yang X, Jia Y: Design of fenofibrate microemulsion for improved bioavailability. Int J Pharm 2011, 420:251-255.

45. Streel $B$, Hubert $P$, Ceccato A: Determination of fenofibric acid in human plasma using automated solid-phase extraction coupled to liquid chromatography. J Chromatogr B Biomed Sci Appl 2000, 742:391-400.

doi:10.1186/s12951-014-0039-3

Cite this article as: Weng et al:: The role of lipid-based nano delivery systems on oral bioavailability enhancement of fenofibrate, a BCS II drug: comparison with fast-release formulations. Journal of Nanobiotechnology 2014 12:39.

\section{Submit your next manuscript to BioMed Central and take full advantage of:}

- Convenient online submission

- Thorough peer review

- No space constraints or color figure charges

- Immediate publication on acceptance

- Inclusion in PubMed, CAS, Scopus and Google Scholar

- Research which is freely available for redistribution 